\title{
Optimal controls for a class of impulsive fractional differential equations with nonlocal conditions
}

\author{
Yu Sun, Haibo Gu* ${ }^{*}$, Yanhui Zhang, Xingru Chen and Xingzhao Wang
}

\section{"Correspondence:}

hbgu_math@163.com

School of Mathematics Sciences, Xinjiang Normal University, Urumqi, China

\begin{abstract}
In this paper, we investigate a class of impulsive fractional differential equations with nonlocal conditions in a Banach space. Firstly, we utilize a fixed point theorem to obtain the existence of solution. Secondly, we derive the sufficient conditions for optimal controls by building approximating minimizing sequences of functions twice.
\end{abstract}

Keywords: Fractional differential equations; Nonlocal conditions; Fractional impulsive conditions; Optimal controls

\section{Introduction}

In recent years, along with multiple phenomena arising in physics, biophysics, engineering, and science (see [1-5] and the references therein), fractional calculus has received increasing attention. The existence of fractional differential equation has received attention of many authors (see [6-9]). The monographs of Bazhlekova [10], Guo et al. [11], Miller et al. [12], Podlubny [13], and the papers [14-16] can provide more details and references about the theory and application of fractional differential equations.

Since the end of the last century, approximate controllability of problems has been paid more and more attention to [17-22]. Impulsive differential equations and optimal conditions have been an active area of research because the impulsive differential system can fully consider the effect of abrupt changes on state. A number of papers extensively study impulsive differential equations with nonlocal differential equations or impulsive conditions only containing integer order derivatives [23, 24]. Kosmatov in [25] considered the nonlinear differential equation initial value problem:

$$
\left\{\begin{array}{l}
{ }^{L} D_{0^{+}}^{\alpha} x(t)=f(t, x(t)), \quad t \in(0,1], t \neq t_{k}, \\
I_{0^{+}}^{1-\alpha} x(0)=x_{0}, \\
{ }^{L} D_{0^{+}}^{\beta} x\left(t_{k}^{+}\right)-{ }^{L} D_{0^{+}}^{\beta} x\left(t_{k}^{-}\right)=J_{k}\left(x\left(t_{k}\right)\right), \quad k=1, \ldots, m,
\end{array}\right.
$$

where $0<\alpha \leq 1,0<\beta<\alpha,{ }^{L} D_{0^{+}}^{\alpha}$ is the Riemann-Liouville derivative of order $\alpha, f$ : $\left([0,1] \backslash\left\{t_{1}, t_{2}, \ldots, t_{m}\right\}\right) \times R \rightarrow R$ is continuous, and $f\left(t_{k} \pm, x\right)$ exists for all $x \in R, k=1, \ldots, m$, $J_{k}\left(x\left(t_{k}\right)\right)$ is a continuous nonlinear map. The existence of solutions of the above nonlinear

(c) The Author(s) 2018. This article is distributed under the terms of the Creative Commons Attribution 4.0 International License (http://creativecommons.org/licenses/by/4.0/), which permits unrestricted use, distribution, and reproduction in any medium, provided you give appropriate credit to the original author(s) and the source, provide a link to the Creative Commons license, and indicate if changes were made. 
differential equation initial value problem was proved by means of Krasnoselskii's theorem in [25].

Motivated by Kosmatov's work, in this paper, we investigate a class of impulsive fractional differential equations with fractional impulsive conditions and nonlocal initial conditions. We firstly prove the existence of solution by the measure of noncompactness and fixed point theorem. Moreover, without ensuring the uniqueness of feasible pairs, we derive a set of sufficient conditions for optimal controls by building approximating minimizing sequences of functions twice. Because the approach is a novelty and the optimal controls for impulsive fractional differential equation with the initial conditions and fractional impulsive conditions are useful in practical application, so the results we obtained are more general than known results.

In this article, we consider the following impulsive fractional differential equation in an abstract space:

$$
\left\{\begin{array}{l}
{ }^{L} D_{0^{+}}^{\alpha} x(t)=f(t, x(t))+B(t) u(t), \quad t \in J^{\prime}=(0, T], t \neq t_{k}, \\
I_{0^{+}}^{1-\alpha} x(0)+g(x)=x_{0}, \quad u \in U_{\mathrm{ad}}, \\
{ }^{L} D_{0^{+}}^{\beta} x\left(t_{k}^{+}\right)-{ }^{L} D_{0^{+}}^{\beta} x\left(t_{k}^{-}\right)=J_{k}\left(x\left(t_{k}\right)\right), \quad k=1, \ldots, m,
\end{array}\right.
$$

where $0<\alpha \leq 1,0<\beta<\alpha, x \in X$, where $X$ is a real Banach space, $f$ is a nonlinear perturbation, $g(x)$ is a given $X$-valued function, $J_{k}\left(x\left(t_{k}\right)\right)$ is a nonlinear map, $U_{\text {ad }}$ is a control set, the control $u \in U_{\text {ad }}$.

The rest of this paper is organized as follows. Section 2 involves some notations and fundamentals of fractional calculus. In Sect. 3, we derive the existence theorem of impulsive fractional differential equations by means of Darbo-Sadovskii's fixed point theorem. In Sect. 4, we establish the optimal controls for a Lagrange problem. An example is given in Sect. 5.

\section{Preliminaries}

In this section, we give some basic definitions and preliminary facts that will be used in the rest of the paper.

Let $X$ be a real Banach space, $£(X)$ be the class of (not necessarily bounded) linear operators in $X$.

The set $C([0, T] ; X)$ is a Banach space of all continuous functions from $[0, T]$ to $X$ with the norm $\|u\|=\sup \{\|u(t)\|, t \in[0, T]\}$.

The set $L^{1}([0, T] ; X)$ is a Banach space of all $X$-valued Bochner integrable functions defined on $[0, T]$ with the norm $\|u\|_{1}=\int_{0}^{T}|u(t)| d t$.

We introduce $P C_{\delta}((0, T] ; X)=\left\{x:(0, T] \rightarrow X: x \in C\left(t_{k}, t_{k+1}\right), k=0, \ldots, m\right.$, such that $x\left(t_{k}^{-}\right), x\left(t_{k}^{+}\right)$exist, $x\left(t_{k}^{-}\right)=x\left(t_{k}\right), k=1, \ldots, m$, and $\lim _{t \rightarrow 0^{+}} t^{\delta} x(t)$ exists $\}$, where $\alpha+\delta-1 \geq 0$ if endowed with the norm $\|x\|_{\delta}=\sup _{t \in(0, T]} t^{\delta}|x(t)| d t, P C_{\delta}$ is also a Banach space.

Definition 2.1 (see [26]) The Riemann-Liouville fractional integral is defined by

$$
I_{0+}^{\alpha} x(t)=j_{\alpha}(t) * u(t)=\int_{0}^{t} j_{\alpha}(t-s) u(s) d s, \quad t>0
$$


where $*$ denotes the convolution,

$$
j_{\alpha}(t)=\frac{t^{\alpha-1}}{\Gamma(\alpha)}
$$

For $x \in C([0, \infty), X)$, the Riemann-Liouville fractional derivative is defined by

$$
{ }^{L} D_{0+}^{\alpha} x(t)=\frac{d}{d t}\left(j_{(1-\alpha)}(t) * u(t)\right)
$$

Now, we introduce the Hausdorff measure of noncompactness $\sigma(\cdot)$ defined on each bounded subset $\Omega$ of the Banach space $X$ by

$$
\sigma(\Omega)=\inf \{\epsilon>0, \Omega \text { has a finite } \epsilon \text {-net in } X\} .
$$

Some basic properties of $\sigma(\cdot)$ are given in the following lemmas.

Lemma 2.2 (see [27]) The measure of noncompactness $\sigma(\cdot)$ satisfies the following:

(i) $\sigma(B)=0$ if and only if $B$ is relatively compact in $X$;

(ii) if $\sigma(\{x\} \cup B)=\sigma(B)$ for every $x \in X$ and every nonempty subset $B \subseteq X$;

(iii) $\sigma(\lambda B) \leq|\lambda| \sigma(B)$ for any $\lambda \in \mathbb{R}$;

(iv) $\sigma\left(B_{1}+B_{2}\right) \leq \sigma\left(B_{1}\right)+\sigma\left(B_{2}\right)$, where $B_{1}+B_{2}=\left\{x+y: x \in B_{1}, y \in B_{2}\right\}$;

(v) $\sigma\left(B_{1} \cup B_{2}\right) \leq \max \left\{\sigma\left(B_{1}\right), \sigma\left(B_{2}\right)\right\}$.

For any $D \subset C([0, T], X)$, we define

$$
\int_{0}^{t} D(s) d s=\left\{\int_{0}^{t} u(s) d s: u \in D\right\} \quad \text { for } t \in[0, T]
$$

where $D(s)=\{u(s) \in X: u \in D\}$.

In order to prove the main result, we introduce the following fixed point theorem.

Lemma 2.3 (Darbo-Sadovskii, see [27]) If $D \subset X$ is a convex bounded and closed set, the continuous mapping $\Lambda: D \rightarrow D$ is a $\sigma$-contraction, then $\Lambda$ has at least one fixed point in $D$.

The set $Y$ is another separable reflexive Banach space where controls $u$ take values. Let $P_{f}(Y) \subset Y$ be nonempty closed and convex. We assume $w:(0, T] \rightarrow P_{f}(Y)$ is a multivalued and measurable mapping, $w(\cdot) \subset E, E \subset Y$ is bounded, the admissible control set $U_{\text {ad }}=$ $S_{w}^{p}=\left\{u \in L^{p}(E) \mid u(t) \in w(t)\right.$, a.e. $\}, p>1$, and $U_{\text {ad }} \neq \emptyset$.

By [25], an important and new equivalent mixed type integral equation for our problem can be established.

Definition 2.4 A function $x \in P C_{\delta}((0, T] ; X)$, whose fractional derivative ${ }^{L} D_{0^{+}}^{\alpha} x(t)$ of order $0<\alpha<1$ exists and is continuous on $(0, T]$ and $t \neq t_{k}$, is said to be a solution of problem (1.1) if it satisfies

$$
\begin{aligned}
x(t)= & \frac{\left(x_{0}-g(x)\right) t^{\alpha-1}}{\Gamma(\alpha)}+\frac{\Gamma(\alpha-\beta)}{\Gamma(\alpha)}\left(\sum_{0<t_{k}<t} t_{k}^{1+\beta-\alpha} J_{k}\left(x\left(t_{k}\right)\right)\right) t^{\alpha-1} \\
& +\frac{1}{\Gamma(\alpha)} \int_{0}^{t}(t-s)^{\alpha-1}(f(s, x(s))+B(s) u(s)) d s .
\end{aligned}
$$




\section{Existence of solution}

In this section, we obtain sufficient conditions for the existence of solutions by utilizing the measure of noncompactness and fixed point theorem.

Let $r$ be a finite positive constant, and set $B_{r}:=\left\{x \in X:\|x\|_{\delta} \leq r\right\}$ and $W_{r}:=\{y \in$ $\left.P C_{\delta}((0, T] ; X): y(t) \in B_{r}, \forall t \in(0, T]\right\}$.

Firstly, we introduce the following hypotheses:

(H1) $f \in C\left((0, T] \backslash\left\{t_{1}, t_{2}, \ldots, t_{m}\right\} \times X\right)$ and $f\left(t_{k} \pm, x\right)$ exist for all $x \in X, k=1, \ldots, m$.

(H2) For a finite positive constant $r>0$, there exists a function $\varphi_{r}$ such that

$$
\|f(t, x)\| \leq \varphi_{r}(t)
$$

for a.e. $t \in(0, T]$ and $x \in B_{r}$, where $I_{0}^{\alpha} \varphi_{r}(t)$ exists and is bounded and continuous.

(H3) For each bounded set $Q \subset X$, there is a constant $q>0$ satisfying

$$
\sigma(f(t, Q)) \leq q \sigma(Q)
$$

where $\sigma$ is the Hausdorff measure of noncompactness.

(H4) $B:(0, T] \rightarrow £(Y, X)$ is an essentially bounded and compact mapping, i.e., $B \in L^{\infty}((0, T], £(Y, X))$.

(H5) $g: P C_{\delta}((0, T] ; X) \rightarrow X$ is a compact and continuous mapping.

(H6) $J_{k}: X \rightarrow X$ satisfies Lipschitz condition with the Lipschitz constants $h_{k}>0$ :

$$
\begin{aligned}
& \left\|J_{k}\left(x_{1}\right)-J_{k}\left(x_{2}\right)\right\| \leq h_{k}\left\|x_{1}-x_{2}\right\|, \quad k=1,2, \ldots, m, \\
& \forall x_{1}, x_{2} \in P C_{\delta}((0, T] ; X) .
\end{aligned}
$$

Remark 3.1 From the definition $U_{\mathrm{ad}}$ and hypothesis (H4), we can easily prove that $B u \in$ $L^{p}((0, T] ; X)$ with $p>1$ for any $u \in U_{\text {ad. }}$. Therefore, $B u \in L^{1}((0, T] ; X)$ and $\|B u\|_{L^{1}}<+\infty$.

Remark 3.2 Under hypothesis (H5), $\sup _{x \in W_{r}}\|g(x)\|<+\infty$.

Theorem 3.1 Suppose that hypotheses $(\mathrm{H} 1)-(\mathrm{H} 6)$ are satisfied, then the impulsive fractional differential equation (1.1) has at least one solution on $(0, T]$ if there is a constant $r>0$ such that

$$
\begin{aligned}
& T^{\alpha+\delta-1} \sup _{x \in W_{r}}\|g(x)\|+T^{\delta} \sup _{t \in(0, T]}\left\{I_{0}^{\alpha}\left|\varphi_{r}(t)\right|\right\} \\
& \quad+T^{\alpha+\delta-1} \Gamma(\alpha-\beta)\left(\sum_{1 \leq k \leq m} t_{k}^{1+\beta-\alpha}\left(\left\|J_{k}(0)\right\|+h_{k} r\right)\right)+\left\|x_{0}\right\|+\frac{T^{\alpha+\delta}\|B u\|_{L^{1}}}{\alpha} \leq r .
\end{aligned}
$$

Proof Let $x_{0} \in X$ be fixed. Define the operator $S$ on $P C_{\delta}((0, T] ; X)$ as follows:

$$
\begin{aligned}
S x(t)= & {\left[\left(x_{0}-g(x)\right)+\Gamma(\alpha-\beta)\left(\sum_{0<t_{k}<t} t_{k}^{1+\beta-\alpha} J_{k}\left(x\left(t_{k}\right)\right)\right)\right] t^{\alpha-1} } \\
& +\int_{0}^{t}(t-s)^{\alpha-1}(f(s, x(s))+B(s) u(s)) d s, \quad t \in(0, T] .
\end{aligned}
$$


The proof process is divided into three steps.

Step I. $S$ maps $W_{r}$ into itself.

It follows from (H1)-(H6) and (3.1), for any $x \in W_{r}$, that we have

$$
\begin{aligned}
\left|t^{\delta} S x(t)\right| \leq & t^{\delta}\left[\left\|\left(x_{0}-g(x)\right)\right\|+\Gamma(\alpha-\beta)\left(\sum_{0<t_{k}<t}\left\|t_{k}^{1+\beta-\alpha} J_{k}\left(x\left(t_{k}\right)\right)\right\|\right)\right] t^{\alpha-1} \\
& +t^{\delta} \int_{0}^{t}\left\|(t-s)^{\alpha-1}(f(s, x(s))+B(s) u(s))\right\| d s \\
\leq & {\left[\left\|x_{0}\right\|+\|g(x)\|+\Gamma(\alpha-\beta)\left(\sum_{1 \leq k \leq m} t_{k}^{1+\beta-\alpha}\left(\left\|J_{k}(0)\right\|+h_{k} r\right)\right)\right] t^{\alpha+\delta-1} } \\
& +t^{\delta} \int_{0}^{t}(t-s)^{\alpha-1}(\|f(s, x(s))\|+\|B(s) u(s)\|) d s \\
\leq & {\left[\left\|x_{0}\right\|+\|g(x)\|+\Gamma(\alpha-\beta)\left(\sum_{1 \leq k \leq m} t_{k}^{1+\beta-\alpha}\left(\left\|J_{k}(0)\right\|+h_{k} r\right)\right)\right] T^{\alpha+\delta-1} } \\
& +t^{\delta} \int_{0}^{t}(t-s)^{\alpha-1}\left|\varphi_{r}(s)\right| d s+t^{\delta}\|B u\|_{L^{1}} \int_{0}^{t}(t-s)^{\alpha-1} d s \\
\leq & {\left[\left\|x_{0}\right\|+\|g(x)\|+\Gamma(\alpha-\beta)\left(\sum_{1 \leq k \leq m} t_{k}^{1+\beta-\alpha}\left(\left\|J_{k}(0)\right\|+h_{k} r\right)\right)\right] T^{\alpha+\delta-1} } \\
& +t^{\delta} I_{0}^{\alpha}\left|\varphi_{r}\right|+t^{\delta}\|B u\|_{L^{1}} \frac{t^{\alpha}}{\alpha} \\
\leq & T^{\alpha+\delta-1} \sup _{x \in W_{r}}\|g(x)\|+T^{\delta} \sup _{t \in(0, T]}\left\{I_{0}^{\alpha}\left|\varphi_{r}(t)\right|\right\} \\
& +T^{\alpha+\delta-1} \Gamma(\alpha-\beta)\left(\sum_{1 \leq k \leq m} t_{k}^{1+\beta-\alpha}\left(\left\|J_{k}(0)\right\|+h_{k} r\right)\right) \\
& +\left\|x_{0}\right\|+\frac{T^{\alpha+\delta}\|B u\|_{L^{1}}}{\alpha} \leq r .
\end{aligned}
$$

Step II. $S$ is continuous in $W_{r}$.

Letting $x_{1}, x_{2} \in W_{r}$, we obtain

$$
\begin{aligned}
& \left|t^{\delta} S x_{1}(t)-t^{\delta} S x_{2}(t)\right| \\
& \leq t^{\delta}\left[\left\|g\left(x_{1}\right)-g\left(x_{2}\right)\right\|+\Gamma(\alpha-\beta)\left(\sum_{0<t_{k}<t} t_{k}^{1+\beta-\alpha}\left\|J_{k}\left(x_{1}\left(t_{k}\right)\right)-J_{k}\left(x_{2}\left(t_{k}\right)\right)\right\|\right)\right] t^{\alpha-1} \\
& \quad+t^{\delta} \int_{0}^{T}(t-s)^{\alpha-1}\left\|f\left(s, x_{1}(s)\right)-f\left(s, x_{2}(s)\right)\right\| d s \\
& \leq\left[\left\|g\left(x_{1}\right)-g\left(x_{2}\right)\right\|+\Gamma(\alpha-\beta)\left(\sum_{0<t_{k}<t} t_{k}^{1+\beta-\alpha}\left\|J_{k}\left(x_{1}\left(t_{k}\right)\right)-J_{k}\left(x_{2}\left(t_{k}\right)\right)\right\|\right)\right] T^{\alpha+\delta-1} \\
& \quad+T^{\delta} \int_{0}^{T}(t-s)^{\alpha-1}\left\|f\left(s, x_{1}(s)\right)-f\left(s, x_{2}(s)\right)\right\| d s .
\end{aligned}
$$

In view of the continuity of $g, J_{k}, f$, we know that $S$ is continuous.

Step III. $S$ has at least one fixed point. 
Set

$$
\begin{aligned}
& I_{1} x(t)=\left(x_{0}-g(x)\right) t^{\alpha-1}, \\
& I_{2} x(t)=\Gamma(\alpha-\beta)\left(\sum_{0<t_{k}<t} t_{k}^{1+\beta-\alpha} J_{k}\left(x\left(t_{k}\right)\right)\right) t^{\alpha-1}, \\
& I_{3} x(t)=\int_{0}^{t}(t-s)^{\alpha-1}(f(s, x(s))+B(s) u(s)) d s
\end{aligned}
$$

for each $x \in W_{r}$. In view of condition (H5), we obtain that the operator $I_{1}$ is compact in $W_{r}$, and so $\sigma\left(I_{1} W_{r}\right)=0$. By $(\mathrm{H} 1)$ and $(\mathrm{H} 3)$, we can derive that $f(t, x(t))$ is a compact mapping in $W_{r}$, for the proof we can refer to Theorem 4.34 in [28]. Combining with (H4), we know that the operator $I_{3}$ is also compact in $W_{r}$, so $\sigma\left(I_{3} W_{r}\right)=0$.

Then, under condition (H6), we notice that $\left\{J_{k}\right\}_{k=1}^{m}$ are Lipschitz continuous. For $x_{1}, x_{2} \in$ $W_{r}$, we have

$$
\begin{aligned}
& \left|t^{\delta} I_{2} x_{1}(t)-t^{\delta} I_{2} x_{2}(t)\right| \\
& \quad \leq t^{\delta} \Gamma(\alpha-\beta)\left(\sum_{0<t_{k}<t} t_{k}^{1+\beta-\alpha}\left\|J_{k}\left(x_{1}\left(t_{k}\right)\right)-J_{k}\left(x_{2}\left(t_{k}\right)\right)\right\|\right) t^{\alpha-1} \\
& \quad \leq \Gamma(\alpha-\beta)\left(\sum_{0<t_{k}<t} t_{k}^{1+\beta-\alpha}\left\|J_{k}\left(x_{1}\left(t_{k}\right)\right)-J_{k}\left(x_{2}\left(t_{k}\right)\right)\right\|\right) T^{\alpha+\delta-1} \\
& \quad \leq T^{\alpha+\delta-1} \Gamma(\alpha-\beta) \sum_{0<t_{k}<t} t_{k}^{1+\beta-\alpha} h_{k}\left\|x_{1}-x_{2}\right\| .
\end{aligned}
$$

Therefore,

$$
\begin{aligned}
& \sigma\left(I_{2} W_{r}\right) \leq T^{\alpha+\delta-1} \Gamma(\alpha-\beta) \sum_{0<t_{k}<t} t_{k}^{1+\beta-\alpha} h_{k} \sigma\left(W_{r}\right), \\
& \sigma\left(S W_{r}\right) \leq \sigma\left(I_{1} W_{r}\right)+\sigma\left(I_{2} W_{r}\right)+\sigma\left(I_{3} W_{r}\right) \leq T^{\alpha+\delta-1} \Gamma(\alpha-\beta)\left(\sum_{0<t_{k}<t} t_{k}^{1+\beta-\alpha} h_{k}\right) \sigma\left(W_{r}\right) .
\end{aligned}
$$

By (3.1) we get

$$
T^{\alpha+\delta-1} \Gamma(\alpha-\beta) \sum_{0<t_{k}<t} t_{k}^{1+\beta-\alpha} h_{k}<1
$$

By Lemma 2.3 we immediately deduce that the operator $S$ has a fixed point in $x \in W_{r}$, i.e., (1.1) has at least one solution. This completes the proof.

Without the Lipschitz continuity of $f$, we cannot obtain the uniqueness of solution of the impulsive fractional differential equation (1.1). Hence, we let $\operatorname{Sol}(u)$ be the space of all solutions of system (1.1) in $W_{r}$ for each $u \in U_{\mathrm{ad}}$.

\section{Optimal controls for problem}

Let $x^{u} \in W_{r}$ denote the solution of system (1.1) associated with the control $u \in U_{\mathrm{ad}}$. 
We discuss the following limited Lagrange problem $(\mathrm{P})$ :

$$
J\left(x^{u}, u\right)=\int_{0}^{T} \psi\left(t, x^{u}(t), u(t)\right) d t .
$$

For all $u \in U_{\text {ad }}$, we can find $x^{0} \in W_{r} \subseteq P C_{\delta}((0, T] ; X)$ and $u^{0} \in U_{\text {ad }}$ such that

$$
J\left(x^{0}, u^{0}\right) \leq J\left(x^{u}, u\right)
$$

where $x^{0} \in W_{r}$ denotes the solution of system (1.1) associated with the control $u^{0} \in U_{\mathrm{ad}}$.

We introduce the following hypotheses:

The function $\psi:(0, T] \times X \times Y$ satisfies the following:

( $\Psi 1)$ The function $\psi:(0, T] \times X \times Y \rightarrow R \cup \infty$ is Borel measurable.

$(\Psi 2) \psi$ is sequentially lower continuous on $X \times Y$ for a.e. $t \in(0, T]$.

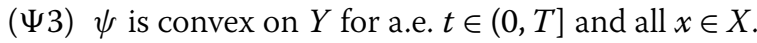

( $\Psi 4)$ There exist two constants $c \geq 0, d>0$ and $\phi \in L^{1}((0, T]: R)$ such that

$$
\psi\left(t, x^{u}(t), u(t)\right) \geq \phi(t)+c\|x\|+d\|u\|_{Y}^{p} .
$$

Remark 4.1 If, for all $x(\cdot) \in W_{r}$, a pair $(x(\cdot), u(\cdot))$ satisfies system (1.1), we say that the pair is feasible. If the pair $\left(x^{u}, u\right)$ is feasible, then $x^{u} \subset W_{r}$.

Theorem 4.1 Suppose that hypotheses $(\Psi 1)-(\Psi 4)$ are satisfied, then problem $(\mathrm{P})$ has at least one optimal feasible pair if it satisfies the condition of Theorem 3.1.

Proof Step I. For each $u \in U_{\mathrm{ad}}$, set

$$
J(u)=\inf _{x^{u} \in \operatorname{Sol}(u)} J\left(x^{u}, u\right) .
$$

If $\operatorname{Sol}(u)$ has infinite elements, obviously we do not have to prove in the case of

$$
J(u)=\inf _{x^{u} \in \operatorname{Sol}(u)} J\left(x^{u}, u\right)=+\infty .
$$

If $\operatorname{Sol}(u)$ has finite elements, there is some $\hat{x}^{u} \in \operatorname{Sol}(u)$ such that $J\left(\hat{x}^{u}, u\right)=\inf _{x^{u} \in \operatorname{Sol}(u)} J\left(x^{u}, u\right)=$ $J(u)$.

We suppose that $J(u)=\inf _{x^{u} \in \operatorname{Sol}(u)} J\left(x^{u}, u\right)<+\infty$. One obtains $J(u)>-\infty$ by conditions $(\Psi 1)-(\Psi 4)$.

In view of the definition of the infimum, we can find a sequence $\left\{x_{n}^{u}\right\}_{n=1}^{\infty} \subseteq \operatorname{Sol}(u)$, which satisfies $J\left(x_{n}^{u}, u\right) \rightarrow J(u)$ as $\rightarrow \infty$.

Since $\left\{\left(x_{n}^{u}, u\right)\right\}_{n=1}^{\infty}$ is a sequence of feasible pairs, one has

$$
\begin{aligned}
x_{n}^{u}(t)= & \left(x_{0}-g\left(x_{n}^{u}\right)\right) t^{\alpha-1}+\Gamma(\alpha-\beta)\left(\sum_{0<t_{k}<t} t_{k}^{1+\beta-\alpha} J_{k}\left(x_{n}^{u}\left(t_{k}\right)\right)\right) t^{\alpha-1} \\
& +\int_{0}^{t}(t-s)^{\alpha-1}\left(f\left(s, x_{n}^{u}(s)\right)+B(s) u(s)\right) d s,
\end{aligned}
$$

where $t \in(0, T]$.

Step II. We can find some $\hat{x}^{u} \in \operatorname{Sol}(u)$ that satisfy $J\left(\hat{x}^{u}, u\right)=\inf _{x^{u} \in \operatorname{Sol}(u)} J\left(x^{u}, u\right)=J(u)$. 
Claim $1\left\{x_{n}^{u}\right\}_{n=1}^{\infty}$ is precompact in $P C_{\delta}((0, T] ; X)$ for all $u \in U_{\mathrm{ad}}$.

For proving the result, we set

$$
x_{n}^{u}=I_{1} x_{n}^{u}+I_{2} x_{n}^{u}+I_{3} x_{n}^{u}
$$

Referring to the proof of Theorem 3.1, we get that $\left\{I_{1} x_{n}^{u}\right\}_{n=1}^{\infty} \subset P C_{\delta}((0, T] ; X)$ and $\left\{I_{3} x_{n}^{u}\right\}_{n=1}^{\infty} \subset P C_{\delta}((0, T] ; X)$ are both precompact. Furthermore, it follows from assumption (H6) that the operator $I_{2}$ is Lipschitz continuous in $P C_{\delta}((0, T] ; X)$, where Lipschitz constant $(T+1)^{\alpha+\delta-1} \Gamma(\alpha-\beta) \sum_{0<t_{k}<t} t_{k}^{1+\beta-\alpha} h_{k}$. Hence, by the given condition and the Hausdorff measure of noncompactness, one has

$$
\begin{aligned}
\rho\left(\left\{x_{n}^{u}\right\}_{n=1}^{\infty}\right) & \leq \rho\left(I_{1}\left\{x_{n}^{u}\right\}_{n=1}^{\infty}\right)+\rho\left(I_{2}\left\{x_{n}^{u}\right\}_{n=1}^{\infty}\right)+\rho\left(I_{3}\left\{x_{n}^{u}\right\}_{n=1}^{\infty}\right) \\
& \leq(T+1)^{\alpha+\delta-1} \Gamma(\alpha-\beta) \sum_{0<t_{k}<t} t_{k}^{1+\beta-\alpha} h_{k} \rho\left(\left\{x_{n}^{u}\right\}_{n=1}^{\infty}\right)
\end{aligned}
$$

Therefore we can conclude that $\rho\left(\left\{x_{n}^{u}\right\}_{n=1}^{\infty}\right)=0$ from the above inequality, so the set $\left\{x_{n}^{u}\right\}_{n=1}^{\infty}$ is precompact in $P C_{\delta}((0, T] ; X)$ for $u \in U_{\mathrm{ad}}$.

Claim $2 \hat{x}^{u} \in \operatorname{Sol}(u)$.

In general, we assume that $x_{n}^{u} \rightarrow \hat{x}^{u}$, as $n \rightarrow \infty$, in $P C_{\delta}((0, T] ; X)$ for $u \in U_{\text {ad }}$. In terms of the dominated convergence theorem and the continuity of $g, J_{i}$, and $f$, when we take the limit $n \rightarrow \infty$ on both sides for (4.1), we obtain

$$
\begin{aligned}
\hat{x}^{u}(t)= & \left(x_{0}-g\left(\hat{x}^{u}\right)\right) t^{\alpha-1}+\Gamma(\alpha-\beta)\left(\sum_{0<t_{k}<t} t_{k}^{1+\beta-\alpha} J_{k}\left(\hat{x}_{n}^{u}\left(t_{k}\right)\right)\right) t^{\alpha-1} \\
& +\int_{0}^{t}(t-s)^{\alpha-1}\left(f\left(s, \hat{x}_{n}^{u}(s)\right)+B(s) u(s)\right) d s
\end{aligned}
$$

where $0 \leq t \leq T$, which shows that $\hat{x}^{u} \in \operatorname{Sol}(u)$.

Step III. $J\left(\hat{x}^{u}, u\right)=\inf _{x^{u} \in \operatorname{Sol}(u)} J\left(x^{u}, u\right)=J(u)$ for all $u \in U_{\mathrm{ad}}$.

According to the Balder theorem and hypotheses $(\Psi 1)-(\Psi 4)$, and using the definition of a feasible pair, we obtain

$$
J(u)=\lim _{n \rightarrow \infty} \int_{0}^{T} \psi\left(t, x_{n}^{u}(t), u(t)\right) d t \geq \int_{0}^{T} \psi\left(t, \hat{x}^{u}(t), u(t)\right) d t=J\left(\hat{x}^{u}, u\right) \geq J(u)
$$

Hence, $J\left(\hat{x}^{u}, u\right)=J(u)$. This implies that $J(u)$ at $\hat{x}^{u} \in P C_{\delta}((0, T] ; X)$ to the minimum for all $u \in U_{\mathrm{ad}}$.

Step IV. There exists $u_{0} \in U_{\text {ad }}$ such that $J\left(u_{0}\right) \leq J(u)$ for $\forall u \in U_{\text {ad }}$.

Suppose that $\inf _{u \in U_{\text {ad }}} J(u)<+\infty$. Referring to the proof of Step I, we deduce that $\inf _{u \in U_{\text {ad }}} J(u)>-\infty$, and there is a sequence $\left\{u_{n}\right\}_{n=1}^{\infty} \subseteq U_{\text {ad }}$ satisfying $J\left(u_{n}\right) \rightarrow \inf _{u \in U_{\text {ad }}} J(u)$ as $n \rightarrow \infty$. We utilize that $L^{p}((0, T] ; Y)$ is a reflexive Banach space and $\left\{u_{n}\right\}_{n=1}^{\infty}$ is bounded in $L^{p}((0, T] ; Y)$. So we can assume that $\left\{u_{n}\right\}_{n=1}^{\infty}$ converges weakly to some $u_{0} \in L^{p}((0, T] ; Y)$ as $n \rightarrow \infty$. 
The set $U_{\mathrm{ad}}$ is convex and closed, so by Mazur's lemma we can deduce that $u_{0} \in U_{\mathrm{ad}}$. For $n \geq 1, \hat{x}^{u_{n}}$ is the solution for (1.1) associated with $u_{n}$, where $J\left(u_{n}\right)$ attains its minimum. Hence the pair $\left(\hat{x}^{u_{n}}, u_{n}\right)$ is feasible, it satisfies the following:

$$
\begin{aligned}
\hat{x}^{u_{n}}(t)= & \left(x_{0}-g\left(\hat{x}^{u_{n}}\right)\right) t^{\alpha-1}+\Gamma(\alpha-\beta)\left(\sum_{0<t_{k}<t} t_{k}^{1+\beta-\alpha} J_{k}\left(\hat{x}^{u_{n}}\left(t_{k}\right)\right)\right) t^{\alpha-1} \\
& +\int_{0}^{t}(t-s)^{\alpha-1}\left(f\left(s, \hat{x}^{u_{n}}(s)\right)+B(s) u(s)\right) d s,
\end{aligned}
$$

where $0 \leq t \leq T$.

Set

$$
\begin{aligned}
& \dot{I}_{3} \hat{x}^{u_{n}}(t)=\int_{0}^{t}(t-s)^{\alpha-1} f\left(s, \hat{x}^{u_{n}}(s)\right) d s, \\
& Z u_{n}(t)=\int_{0}^{t}(t-s)^{\alpha-1} B(s) u_{n}(s) d s .
\end{aligned}
$$

We have

$$
\hat{x}^{u_{n}}(t)=I_{1} \hat{x}^{u_{n}}(t)+I_{2} \hat{x}^{u_{n}}(t)+\dot{I}_{3} \hat{x}^{u_{n}}(t)+Z u_{n}(t),
$$

where $0 \leq t \leq T$.

We know that $\left\{I_{1} \hat{x}^{u_{n}}\right\}_{n=1}^{\infty}$ and $Z u_{n}(t)$ are both precompact in $P C_{\delta}((0, T] ; X)$ and $I_{2}$ satisfies the Lipschitz continuity in $P C_{\delta}((0, T] ; X)$ with the Lipschitz constant

$$
(T+1)^{\alpha+\delta-1} \Gamma(\alpha-\beta) \sum_{0<t_{k}<t} t_{k}^{1+\beta-\alpha} h_{k}<1 .
$$

Moreover, in view of given assumptions, we can see that $\left\{I_{3} \hat{x}^{u_{n}}\right\}_{n=1}^{\infty} \subseteq P C_{\delta}((0, T] ; X)$ is precompact.

Referring to the proof of Theorem 3.1, we imply that $\rho\left(\left\{\hat{x}^{u_{n}}\right\}_{n=1}^{\infty}\right)=0$, i.e., $\left\{\hat{x}^{u_{n}}\right\}_{n=1}^{\infty} \subseteq$ $P C_{\delta}((0, T] ; X)$ is precompact. Thus we can find a subsequence which is connected with $\hat{x}^{u_{0}} \in P C_{\delta}((0, T] ; X)$ and $\left\{\hat{x}^{u_{n}}\right\}_{n=1}^{\infty}$ satisfying that $\hat{x}^{u_{n}} \rightarrow \hat{x}^{u_{0}}$, as $n \rightarrow \infty$ in $P C_{\delta}((0, T] ; X)$. When we take the limit $n \rightarrow \infty$ on both sides for (4.2), we get

$$
\begin{aligned}
\hat{x}^{u_{0}}(t)= & \left(x_{0}-g\left(\hat{x}^{u_{0}}\right)\right) t^{\alpha-1}+\Gamma(\alpha-\beta)\left(\sum_{0<t_{k}<t} t_{k}^{1+\beta-\alpha} J_{k}\left(\hat{x}^{u_{0}}\left(t_{k}\right)\right)\right) t^{\alpha-1} \\
& +\int_{0}^{t}(t-s)^{\alpha-1}\left(f\left(s, \hat{x}^{u_{0}}(s)\right)+B(s) u(s)\right) d s,
\end{aligned}
$$

where $0 \leq t \leq T$. Therefore the pair $\left(\hat{x}^{u}, u_{0}\right)$ is feasible.

In view of the Balder theorem and assumptions $(\Psi 1)-(\Psi 4)$, we have

$$
\begin{aligned}
\inf _{u \in U_{\mathrm{ad}}} J(u) & =\lim _{n \rightarrow \infty} \int_{0}^{T} \psi\left(t, \hat{x}^{u_{n}}(t), u_{n}(t)\right) d t \geq \int_{0}^{T} \psi\left(t, \hat{x}^{u_{0}}(t), u_{0}(t)\right) d t \\
& =J\left(\hat{x}^{u_{0}}, u_{0}\right) \geq \inf _{u \in U_{\mathrm{ad}}} J(u) .
\end{aligned}
$$


Thus,

$$
J\left(\hat{x}^{u_{0}}, u_{0}\right)=J\left(u_{0}\right)=\inf _{x^{u_{0}} \in \operatorname{Sol}\left(u_{0}\right)} J\left(x^{u_{0}}, u_{0}\right) .
$$

Furthermore,

$$
J\left(u_{0}\right)=\inf _{u \in U_{\mathrm{ad}}} J(u)
$$

i.e., $J$ at $u_{0} \in U_{\text {ad }}$ to the minimum.

\section{An example}

Example 5.1 Consider the following impulsive fractional differential equations:

$$
\left\{\begin{array}{l}
{ }^{L} D_{0^{+}}^{\alpha} x(t)=\frac{e^{-t}|x(t)|}{\left(1+e^{t}\right)(1+|x(t)|)}+u(t), \quad t \in J^{\prime}=(0,1], t \neq t_{1}, \\
I_{0^{+}}^{1-\alpha} x(0)=g(x) \\
{ }^{L} D_{0^{+}}^{\beta} x\left(t_{1}^{+}\right)-{ }^{L} D_{0^{+}}^{\beta} x\left(t_{1}^{-}\right)=\frac{\left|x\left(t_{1}\right)\right|}{3+\left|x\left(t_{1}\right)\right|} .
\end{array}\right.
$$

We take

$$
\begin{aligned}
& f:(0,1] \times R \rightarrow R \quad \text { and } \quad f(t, x)=\frac{e^{-t}|x|}{\left(1+e^{t}\right)(1+|x|)} \\
& g: P C_{\delta}((0, T] ; X) \rightarrow X \quad \text { and } g(x) \text { is a continuous and compact map, } \\
& J_{1}: X \rightarrow X \text { and } J_{1}(x)=\frac{|x|}{3+|x|}
\end{aligned}
$$

Let $x_{1}, x_{2} \in B_{r}$ and $t \in(0,1]$, we have

$$
\begin{aligned}
\left|f\left(t, x_{1}\right)-f\left(t, x_{2}\right)\right| & =\frac{e^{-t}}{\left(1+e^{t}\right)}\left|\frac{\left|x_{1}\right|}{1+\left|x_{1}\right|}-\frac{\left|x_{2}\right|}{1+\left|x_{2}\right|}\right|=\frac{e^{-t}|| x_{1}|-| x_{2}||}{\left(1+e^{t}\right)\left(1+\left|x_{1}\right|\right)\left(1+\left|x_{2}\right|\right)} \\
& \leq \frac{e^{-t}}{1+e^{t}}\left|x_{1}-x_{2}\right| \leq \frac{e^{-t}}{2}\left|x_{1}-x_{2}\right| .
\end{aligned}
$$

Obviously, for each $x_{1}, x_{2} \in B_{r}$ and $t \in(0,1]$,

$$
\begin{aligned}
& |f(t, x)|=\frac{e^{-t}}{1+e^{t}} \frac{|x|}{1+|x|} \leq \frac{e^{-t}}{1+e^{t}} \leq \frac{e^{-t}}{2} . \\
& \left|J_{1}\left(x_{1}\right)-J_{1}\left(x_{2}\right)\right| \leq \frac{1}{3}\left|x_{1}-x_{2}\right| .
\end{aligned}
$$

So hypotheses (H1)-(H6) are satisfied.

We define the cost function $J\left(x^{u}, u\right)=\int_{0}^{1}\left(\left\|x^{u}(t)\right\|_{X}^{2}+\|u(t)\|_{Y}^{2}\right) d t$, where $\left(x^{u}, u\right)$ is a feasible pair. If the inequality

$$
\begin{aligned}
& T^{\alpha+\delta-1} \sup _{x \in W_{r}}\|g(x)\|+T^{\delta} \sup _{t \in(0,1]}\left\{I_{0}^{\alpha}\left|\frac{e^{-t}}{2}\right|\right\}+\frac{r}{3} T^{\alpha+\delta-1} \Gamma(\alpha-\beta) t_{1}^{1+\beta-\alpha} \\
& +\left\|x_{0}\right\|+\frac{T^{\alpha+\delta}\|B u\|_{L^{1}}}{\alpha} \leq r
\end{aligned}
$$


holds, so all the conditions in Theorem 3.1 are satisfied, our results can be applied to problem (5.1).

\section{Conclusions}

In this paper, we have considered the existence of solution and optimal controls for a class of impulsive fractional differential equations with fractional impulsive conditions and nonlocal conditions. By constructing operator $S$, using the measure of noncompactness method and fixed point theorem, we derived some sufficient assumptions to guarantee the existence of solution. By constructing approximating minimizing sequences of functions twice, we also obtained the optimal controls of impulsive fractional differential equations. Our results are more general than known results. Based on the results of this paper, we will investigate the impulsive fractional semilinear differential equations in the next paper.

\section{Acknowledgements}

This work is supported by Xinjiang Normal University Doctoral Research Fund (XJNUB1540), Graduate Science and Technology Innovation Project of Xinjiang Normal University (XSY201702001), Science and Technology Innovation Personnel Training Project for Youth of Xinjiang (QN2016JQ0367), Mathematics Key Subject of Xinjiang Normal University (17SDKD1 106). The authors would like to thank the referees for their careful reading of this paper.

\section{Competing interests}

The authors declare that they have no competing interest.

\section{Authors' contributions}

All authors read and approved the final manuscript.

\section{Publisher's Note}

Springer Nature remains neutral with regard to jurisdictional claims in published maps and institutional affiliations.

Received: 16 January 2018 Accepted: 1 April 2018 Published online: 06 April 2018

\section{References}

1. Eidelman, S.D., Kochubei, A.N.: Cauchy problem for fractional diffusion equations. J. Differ. Equ. 199, $211-255$ (2004)

2. Hilfer, R.: Applications of Fractional Calculus in Physics. World Scientific, Singapore (2000)

3. Sandev, T., Metzler, R., Tomovski, Ž.: Fractional diffusion equation with a generalized Riemann-Liouville time fractional derivative. J. Phys. A, Math. Theor. 44, 255203 (2011)

4. Yan, J.P., Li, C.P.: On chaos synchronization of fractional differential equations. Chaos Solitons Fractals 32, 725-735 (2007)

5. Yang, H., Luo, G., Karnchanaphanurach, P., Louie, T., Rech, I., Cova, S., Xun, L., Xie, X.: Protein conformational dynamics probed by single-molecule electron transfer. Science 302, 262-266 (2003)

6. Agarwal, R.P., Benchohra, M., Slimani, B.A.: Existence results for differential equations with fractional order and impulses. Mem. Differ. Equ. Math. Phys. 44, 1-21 (2008)

7. Ahmad, B., Nieto, J.J.: Existence results for nonlinear boundary value problems of fractional integrodifferential equations with integral boundary conditions. Bound. Value Probl. 2009, 708576 (2009)

8. Tin, Y., Bai, Z.: Existence results for the three-point impulsive boundary value problem involving fractional differential equations. Comput. Math. Appl. 59, 2601-2609 (2010)

9. Zhang, X., Huang, X., Lou, Z.: The existence and uniqueness of mild solutions for impulsive fractional equations with nonlocal conditions and infinite delay. Nonlinear Anal. Hybrid Syst. 4, 775-781 (2010)

10. Bazhlekova, E.: Fractional evolution equations in Banach space. Ph.D. thesis, Eindhoven University of Technology (2001)

11. Guo, D.J., Lakshmikantham, V., Liu, X.Z.: Nonlinear Integral Equations in Abstract Spaces. Kluwer Academic, Dordrecht (1996)

12. Miller, K.S., Ross, B.: An Introduction to the Fractional Calculus and Differential Equations. Wiley, New York (1993)

13. Podlubny, I.: Fractional Differential Equations. Academic Press, San Diego (1999)

14. Agarwal, R.P., Lakshmikantham, V., Nieto, J.J.: On the concept of solution for fractional differential equations with uncertainty. Nonlinear Anal. TMA 72, 2859-2862 (2010)

15. Kilbas, A.A., Srivastava, H.M., Trujillo, J.J.: Theory an Application of Fractional Differential Equations. Elsevier, Amsterdam (2006)

16. Lakshmikantham, V., Vatsala, A.S.: Basic theory of fractional differential equations. Nonlinear Anal. 69, 2677-2682 (2008)

17. Ganesh, R., Sakthivel, R., Mahmudov, N.I.: Approximate controllability of fractional functional equations with infinite delay. Topol. Methods Nonlinear Anal. 43, 345-364 (2014)

18. Mahmudov, N.I.: Approximate controllability of evolution systems with nonlocal conditions. Nonlinear Anal. 68 536-546 (2008) 
19. Park, J.Y., Kang, S.N.: Approximate controllability of neutral functional differential system with unbounded delay. Int. J. Math. Math. Sci. 26, 737-744 (2001)

20. Sakthivel, R., Mahmudov, N.I., Kim, J.H.: Approximate controllability of nonlinear impulsive differential systems. Rep. Math. Phys. 60, 85-96 (2007)

21. Sakthivel, R., Ren, Y., Debbouche, A., Mahmudov, N.I: Approximate controllability of fractional stochastic differential inclusions with nonlocal conditions. Appl. Anal. 95, 2361-2382 (2016)

22. Wan, X., Sun, J.: Approximate controllability for abstract measure differential systems. Syst. Control Lett. $61,50-54$ (2012)

23. Benchohra, M., Henderson, J., Ntouyas, S.: Impulsive Differential Equations and Inclusions. Hindawi Publishing Corporation, New York (2006)

24. Lakshmikantham, V., Bainov, D.D., Simeonov, P.S.: Theory of Impulsive Differential Equations. Series in Modern Applied Mathematics. World Scientific, Singapore (1994)

25. Kosmatov, N.: Initial value problems of fractional order with fractional impulsive conditions. Results Math. 63 , 1289-1310 (2013)

26. Zhou, Y.: Attractivity for fractional differential equations in Banach space. Appl. Math. 75, 1-6 (2018)

27. Banas̀, J., Goebel, K.: Measure of Noncompactness in Banach Space. Dekker, New York (1980)

28. Zhou, Y.: Basic Theory of Fractional Differential Equations. World Scientific, Singapore (2014)

\section{Submit your manuscript to a SpringerOpen ${ }^{\circ}$} journal and benefit from:

- Convenient online submission

- Rigorous peer review

- Open access: articles freely available online

- High visibility within the field

- Retaining the copyright to your article

Submit your next manuscript at $\gg$ springeropen.com 